\title{
RA synovium harbours distinct fibroblast subsets
}

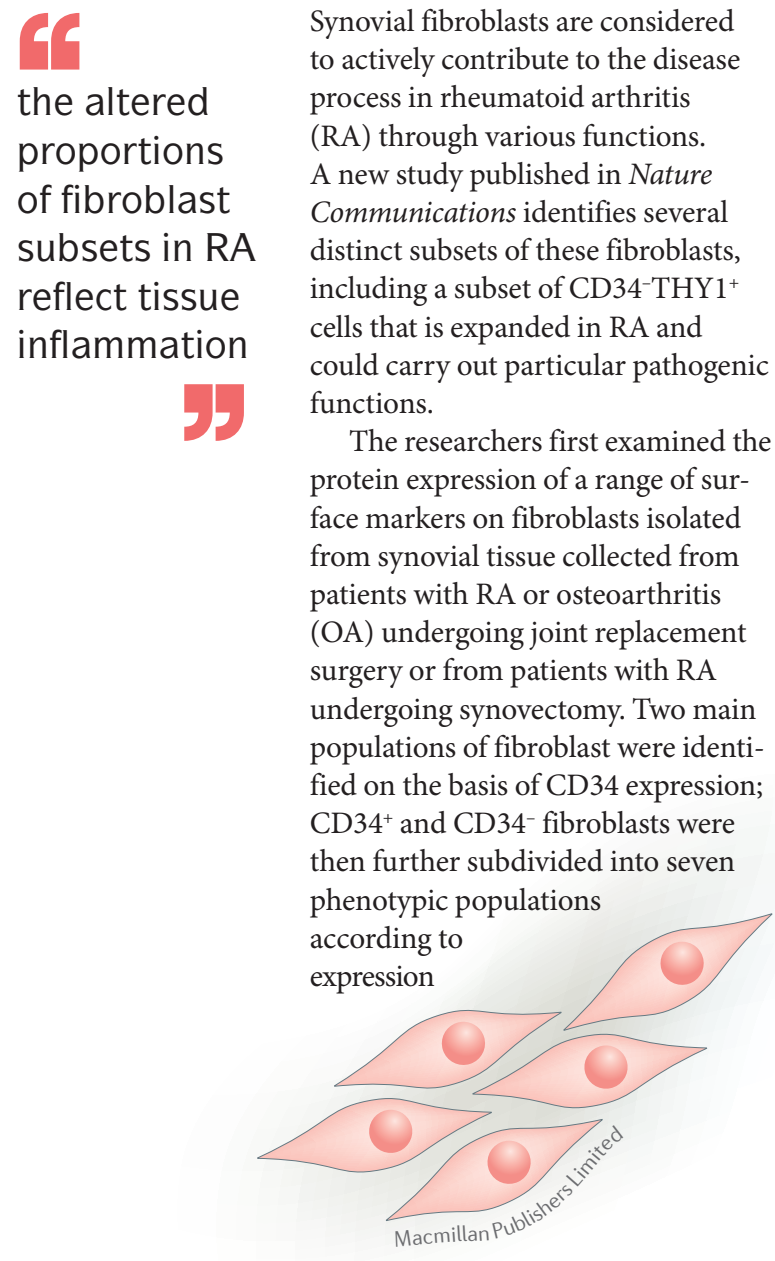

of THY1 (also known as CD90) and cadherin 11 (CDH11). These seven gated populations also had distinct gene expression profiles in both microarray and RNA-seq data sets. The cells were grouped into three major subsets - CD34-THY1-, CD $34^{-} \mathrm{THY}^{+}$and $\mathrm{CD} 34^{+}$fibroblasts - using principal component analysis (PCA) of the microarray data; interestingly, unbiased clustering of fibroblasts by single-cell RNA-seq confirmed these three subsets.

The proportions of the fibroblast subsets differed between synovial tissue from patients with RA and that from patients with OA, with CD $34^{-} \mathrm{THY}^{+}$cells in particular being threefold more abundant in RA than in OA (22\% versus $8 \%$ ). In biopsy-obtained synovial tissue from the knees of patients with RA, the proportion of $\mathrm{CD} 34^{-} \mathrm{THY}^{+}$ fibroblasts correlated with the proportion of infiltrating leukocytes by flow cytometry, as well as with histological synovitis and synovial hypertrophy as assessed by use of ultrasonography. These findings suggest that the altered proportions of fibroblast subsets in RA reflect tissue inflammation. No correlation was found between the proportion of CD34-THY1+ fibroblasts and disease duration (as measured in years).

In RA tissue, CD34-THY1+ fibroblasts localized around blood vessels in the sublining area of the synovium. Notably, most of the CD34-THY1 ${ }^{+}$ fibroblast population in RA tissue were also positive for $\mathrm{CDH} 11$, the expression of which is characteristic of fibroblasts and has been linked to the pathological activity of these cells. Moreover, the transcriptomic profile of CD34-THY1+ fibroblasts correlated with cellular functions in vitro, including invasion and migratory behaviour, secretion of pro-inflammatory cytokines and proliferation.

Together, the findings suggest that distinct fibroblast subsets perform different molecular functions in RA. The researchers suggest that the identification of pathogenic fibroblast subsets could reveal new therapeutic targets.

Sarah Onuora

ORIGINAL ARTICLE Mizoguchi, M. et al.

Functionally distinct disease-associated fibroblast subsets in rheumatoid arthritis. Nat. Commun. $\mathbf{9}$, 789 (2018) 